\title{
Secular Slowing of Auditory Simple Reaction Time in Sweden (1959-1985)
}

\author{
Guy Madison ${ }^{*}$, Michael A. Woodley of Menie ${ }^{2,3}$ and Justus Sänger ${ }^{2}$ \\ ${ }^{1}$ Department of Psychology, Umeå University, Umeå, Sweden, ${ }^{2}$ Department of Psychology, Technische Universität \\ Chemnitz, Chemnitz, Germany, ${ }^{3}$ Center Leo Apostel for Interdisciplinary Studies, Vrije Universiteit Brussel, Brussels, Belgium
}

\section{OPEN ACCESS}

Edited by:

Juliana Yordanova,

Bulgarian Academy of Sciences,

Bulgaria

Reviewed by:

Björn Albrecht,

University of Göttingen, Germany

Soledad Ballesteros,

National University of Distance

Education, Spain

${ }^{*}$ Correspondence:

Guy Madison

guy.madison@umu.se

Received: 06 February 2016 Accepted: 02 August 2016

Published: 18 August 2016

Citation:

Madison G, Woodley of Menie MA and Sänger J (2016) Secular Slowing

of Auditory Simple Reaction Time

in Sweden (1959-1985).

Front. Hum. Neurosci. 10:407.

doi: 10.3389/fnhum.2016.00407
There are indications that simple reaction time might have slowed in Western populations, based on both cohort- and multi-study comparisons. A possible limitation of the latter method in particular is measurement error stemming from methods variance, which results from the fact that instruments and experimental conditions change over time and between studies. We therefore set out to measure the simple auditory reaction time (SRT) of 7,081 individuals (2,997 males and 4,084 females) born in Sweden 1959-1985 (subjects were aged between 27 and 54 years at time of measurement). Depending on age cut-offs and adjustment for aging related slowing of SRT, the data indicate that SRT has increased by between 3 and $16 \mathrm{~ms}$ in the 27 birth years covered in the present sample. This slowing is unlikely to be explained by attrition, which was evaluated by comparing the general intelligence $\times$ birth-year interactions and standard deviations for both male participants and dropouts, utilizing military conscript cognitive ability data. The present result is consistent with previous studies employing alternative methods, and may indicate the operation of several synergistic factors, such as recent micro-evolutionary trends favoring lower $g$ in Sweden and the effects of industrially produced neurotoxic substances on peripheral nerve conduction velocity.

Keywords: reaction time, intelligence, secular trend, auditory reaction time, evolution, human evolution

\section{INTRODUCTION}

It has recently been suggested that physiological measures of cognitive ability, such as simple reaction time (SRT), may be immune to the Flynn effect - the increase in psychometric intelligence (IQ) of three points per decade (Pietschnig and Voracek, 2015). This results from the fact that such measures serve as biomarkers of the underlying general intelligence factor $(g)$ and are therefore capable of consistently measuring the same cognitive parameter across cohorts, i.e., processing speed (Jensen, 2011). In contrast, pencil-and-paper tests of IQ fail to precisely and invariantly measure the same dimension of performance across cohorts. It is this lack of test invariance that is thought to in part give rise to the Flynn effect, which is characterized by heterogeneous gains among test specificities rather than in substantive latent factors, such as $g$ (Wicherts et al., 2004). The secular trend characteristics of SRT and other elementary cognitive tasks (ECTs) are therefore of considerable theoretical interest, as they may in some cases reveal important information about the secular trend characteristics of $g$ (Gottfredson, 2007).

Various lines of evidence support the argument that secular trends on simple ECT performance may in fact be quite discrepant from the Flynn effect on conventional psychometric tests. For 
example, Nettelbeck and Wilson (2004) found no indication of secular gains on inspection time performance over 30 years in an Australian sample, despite indications of concurrent gains on a test of verbal ability administered to the same cohorts. Simple timing tasks (i.e., finger tapping) similarly reveal no Flynn effect, despite concurrent gains on the Trail Making and the Symbol Digit tests (Dickinson and Hiscock, 2011). A metaanalytic study by Silverman (2010) revealed apparent long term declines in performance on visual simple reaction time (SRT) across a handful of Western countries between the 1880s and the 2000s. Woodley et al. (2013) reanalyzed Silverman's data utilizing meta-regression, finding evidence of a robust secular trend toward slowing SRT between the years 1889 and 2004, amounting to a lag increase of $77 \mathrm{~ms}$. This finding was met with such skepticism that six commentaries advancing a range of criticisms were published in response (Dodonova and Dodonov, 2013; Flynn, 2013; Silverman, 2013; Nettelbeck, 2014; Parker, 2014; Woods et al., 2015). A common theme among these commentaries concerned the possibility of confounding effects stemming from the presence of uncontrolled methods variance, i.e., variation in the results of studies due to variations in the instruments and in the experimental protocols used to measure SRT (Jensen, 2006, 2011). Dodonova and Dodonov (2013) even estimated an alternative and shallower secular trend in SRT based on a set of corrections for method-related increases in the measured SRTs. A different application of these corrections, coupled with a tightening of the inclusion rules so as to exclude non-Anglosphere data points from consideration, established the presence of secular slowing trends amounting to between $-34.49 \mathrm{~ms}$ and $-44.6 \mathrm{~ms}$ across 115 years, but with much improved fit, as indicated by lower heterogeneity among the studies (Woodley et al., 2014b). Much controversy surrounded the use of Galton's nineteenth century population SRT values, which were confounded by a small calibration error in the chronoscope and also possibly by his selecting only the fastest of three trials per participant. On this basis Dodonova and Dodonov (2013) proposed that Galton's sample might have had a mean SRT of around $207 \mathrm{~ms}$, rather than $188 \mathrm{~ms}$. Thresholding analysis revealed however that Galton's sample could have been slower still $(213 \mathrm{~ms})$, and the secular decline trend detected in Woodley et al. (2014b) would still hold (Woodley of Menie et al., 2015a). A more recent reanalysis of the Silverman data in which only four large and representative UK studies (Ns > 500) were corrected for methods variance, and in which Galton's sample was assigned a mean SRT of $208.5 \mathrm{~ms}$, revealed a secular slowing amounting to $-22.8 \mathrm{~ms}$ across a century (Woodley of Menie et al., 2015b). A similar methods variance corrected crossstudy comparison involving four normalization samples indicates secular performance declines in another ECT - color acuity (Woodley of Menie and Fernandes, 2016).

Despite the apparent robustness of the results of Silverman's original study, Dodonova and Dodonov's (2013) criticisms nonetheless highlighted the importance of a lack of standardization in methods as a genuine source of uncertainty in estimating secular changes in SRT using cross-study comparisons. One solution to this problem would be to record SRTs with common instrumentation and methods from age-matched cohorts over the course of at least a few decades, obviously a major undertaking. While such data exist for inspection times (Nettelbeck and Wilson, 2004) and finger tapping (Dickinson and Hiscock, 2011), to the best of our knowledge no such data yet exist for SRT.

Another solution is to obtain both cross-sectional and longitudinal SRT data from participants covering a wide age range. The ratio of the two trends can be used to infer the presence of secular trends on the basis that steeper longitudinal relative to cross-sectional slowing trends would indicate that older cohorts were relative faster than younger ones when matched for age, suggesting a secular decline in performance, and vice-versa in the case of secular gains (Verhaeghen, 2014). Based on an analysis of nine studies reporting both cross-sectional and longitudinal change data for a variety of processing speed measures, Verhaeghen (2014) concludes that the complexity of the measure seems to increase the sensitivity of the measure to secular gains. A more complex processing speed measure such as perceptual speed is, for example, associated with ratios $>1$, indicating that the longitudinal trend is shallower than the crosssectional trend, and that younger cohorts are relatively faster than older ones when matched for age based on trend extrapolation. By contrast simple measures of processing speed (such as Go/No Go) are associated with ratios $<1$, indicating potential secular declines. In a reanalysis of Verhaeghen's (2014) data on SRT, it was found that the weighted average ratio across three studies was 0.90 (Woodley of Menie et al., 2015b). Verhaeghen (2014, p. 256) himself reports combined ratios of 0.85 for SRT and 0.97 for choice RT. In any case, these findings are consistent with modest SRT, and possibly also CRT latency increases across generations. A reanalysis of the study of Deary and Der (2005), utilizing a curve-fitting variant of Verhaeghen's method, found evidence of significant secular declines among the female, but not the male cohorts (Woodley et al., 2014a). As more complex processing speed measures are multi-factorial (they tap decision time and certain memory and attention components in addition to pure processing speed; Jensen, 2006), improvements with respect to these other, potentially more trainable performance determinants may mask secular declines at the level of processing speed on such measures, which might account for Verhaeghen's (2014) observation of a positive association between ECT measure complexity and Flynn effect sensitivity.

Large-scale cross-sectional studies investigating measures of SRT have found that age related slowing is not homogeneous across the range of ages, with performance typically decreasing more rapidly among older cohorts. For example, Jaworski et al. (2011) found no significant aging effects on auditory SRT when the mean of a group of 21- to 35-year-olds were compared with a group of 36- to 55-year-olds, with the differences becoming much more pronounced and significant when both of the groups were compared with a group of 56- to 80-year-olds. Similar observations have been made by Der and Deary (2006, p. 65) using cross-sectional comparison of performance on visual SRT, with performance among those younger than 50 showing little variation as a function of age, and sharp performance declines occurring among those older than 50. One explanation for this heterogeneity, consistent with Verhaeghen's (2014) results, 
is that period effects (i.e., in this case secular slowing in the speed of SRT) are canceling out the longitudinal age-related slowing among the younger cohorts. In other words people in their 40s are now approximately as slow as people in their 20s after experiencing age-related slowing, indicating that they were relatively faster when younger. Older cohorts (i.e., those $>50$ years of age) may experience much more pronounced agerelated slowing (e.g., Deary and Der, 2005), which offsets the period effect, yielding an apparent cross-sectional decline in performance among this group. Directly adjusting cross-sectional data for the longitudinal trend might therefore bring out the secular slowing trend.

To investigate this possibility, data on auditory SRT from 7,081 Swedish individuals born between 1959 and 1985 were collected in connection with a large web-based data collection that included a wide range of instruments and questionnaires. The cross-sectional data were adjusted for longitudinal agerelated auditory SRT slowing using estimates derived from Fozard et al. (1994), in order to establish the presence of a secular trend. Remarkably, however, even the non-adjusted SRT data revealed clear indications of increasing SRT performance with age among the cohorts aged 27-50, which is the range that appears to be 'static' in terms of slowing in cross-sectional studies of SRT performance (e.g., Der and Deary, 2006; Jaworski et al., 2011). On this basis it was decided to present both aging-adjusted and non-adjusted SRT data.

\section{MATERIALS AND METHODS}

\section{Participants}

Data were collected online from October 2012 to May 2013 from the STAGE cohort of twins born between 1959 and 1985 (Lichtenstein et al., 2006). This cohort is part of the Swedish Twin Registry (STR), and has been involved in one previous data collection wave that took place in 2005-2006. The STR includes all twins born in Sweden since 1886 (Lichtenstein et al., 2002 and Lichtenstein et al., 2006 for further details on the STAGE cohort and its representativity). The study was approved by the Regional Ethics Review Board in Stockholm (Dnr 2011/570-31/5, 2011/1425-31, and 2012/1107/32). We approached all 32,005 twins in the cohort with a letter sent to their residential addresses, which contained a brief description of the study, a statement that their participation was voluntary and may be discontinued at any time, and that their commencing the web survey would constitute giving informed consent. The letter contained an individual pass code that the participants used to log in to the web survey. The full sample that completed the present wave consisted of 11,543 twins, 5,651 singletons and 5,892 that were part of a complete twin pair, comprised of 6,651 females and 4,892 males, with ages between 27 and 54 at the time of responding to the survey $(M=40.7, S D=7.75)$.

\section{Variables}

The survey contained a wide range of instruments and questions that took between 55 and $90 \mathrm{~min}$ to complete, the procedures of which are further described in Appendix 1.3. Many of these instruments are described in previous publications (e.g., Mosing et al., 2014a,b, 2015; Ullén et al., 2014, 2015; Theorell et al., 2015). Of these, only auditory SRT, sex, and birth year were utilized in the present study.

The auditory SRT task was implemented both as a Flash and as a Shockwave application, as detailed in Appendix 1.1. Auditory stimulation was chosen because the control and latency of sound presentation varies less across different computer systems than visual presentation. Shockwave had superior temporal accuracy and was used if it was installed on the participant's computer. Otherwise, the participant was given the option of installing Shockwave. If the participant declined or the Shockwave installation failed, the Flash application was used. If Flash was not installed and the participant did not agree to install it, no SRT data could be obtained and that participant was excluded from the analysis. The SRT datum for each participant was the median of 25 trials, corrected for known differences between Flash and Shockwave, different browsers, operating systems, and so forth, in order to increase inter-individual reliability as described in Appendix 1.2.

Conscript intelligence test scores were obtained from mandatory military testing that the males in the present study underwent when they were about 19 years old. The purpose of obtaining these was to assess possible effects of cognition on attrition, specifically if there was any cognitive ability $\times$ birth year interaction that might be an alternative explanation for secular changes in the SRT measure. The nature of these military intelligence tests are secret, so as to avoid their dissemination and hence cheating or other validity threats. The authority releases no information about the items, but does release the maximum score and the raw scores for each individual. Test results were only available for the subset of the male cohort born between 1959 and 1976, because systematic military psychometric testing was gradually discontinued in the late 1990s.

\section{RESULTS}

Of the total 32,005 twins that were invited to participate, 11,543 logged in and responded to at least one item after receiving up to three surface mail reminders, and 7,081 completed the SRT tests. First, intra-individual SRT reliability was investigated by correlating the medians for the first 12 and the second 13 SRTs for each participant. As this value was as high as 0.953 we did not attempt to correct for it in the following analyses. Table 1 shows the descriptive statistics of the SRT data.

TABLE 1 | Descriptive data for SRT, 1959-1985.

\begin{tabular}{lccc}
\hline & \multicolumn{3}{c}{ SRT } \\
\cline { 2 - 4 } Sex & $\boldsymbol{N}$ & $\boldsymbol{M}$ & SD \\
\hline Females & 4,084 & 243.9 & 47.5 \\
Males & 2,997 & 239.5 & 48.4 \\
Both & 7,081 & 241.0 & 47.1
\end{tabular}


TABLE 2 | Regressions of auditory SRT on birth year for 1959-1985 and 1963-1985, for aging-adjusted and non-adjusted SRTs, and for females, males, and both sexes together.

\begin{tabular}{|c|c|c|c|c|c|c|c|}
\hline Year range & $N$ & Intercept & Slope & SE & $T$ & $R^{2}$ & $p$ \\
\hline \multicolumn{8}{|c|}{ Non-adjusted data, both sexes } \\
\hline 1959-1985 & 7,081 & 163 & 0.040 & 0.073 & 0.54 & $<0.001$ & 0.580 \\
\hline 1963-1985 & 5,966 & -220 & 0.233 & 0.091 & 2.57 & 0.001 & $<0.01$ \\
\hline \multicolumn{8}{|c|}{ Aging-adjusted data, both sexes } \\
\hline 1959-1985 & 7,081 & -828 & 0.539 & 0.073 & 7.41 & 0.008 & $<0.00001$ \\
\hline 1963-1985 & 5,966 & -1212 & 0.730 & 0.091 & 8.07 & 0.011 & $<0.00001$ \\
\hline \multicolumn{8}{|c|}{ Non-adjusted data, females } \\
\hline 1959-1985 & 4,084 & 284 & 0.021 & 0.095 & 0.22 & $<0.001$ & 0.826 \\
\hline 1963-1985 & 3,427 & -10.6 & 0.128 & 0.118 & 1.08 & $<0.001$ & 0.278 \\
\hline \multicolumn{8}{|c|}{ Aging-adjusted data, females } \\
\hline 1959-1985 & 4,084 & -708 & 0.479 & 0.095 & 5.05 & 0.006 & $<0.00001$ \\
\hline 1963-1985 & 3,427 & -1003 & 0.630 & 0.118 & 5.31 & 0.008 & $<0.00001$ \\
\hline \multicolumn{8}{|c|}{ Non-adjusted data, males } \\
\hline 1959-1985 & 2,997 & -11.2 & 0.127 & 0.113 & 1.12 & $<0.001$ & 0.262 \\
\hline 1963-1985 & 2,539 & -503 & 0.375 & 0.141 & 2.66 & 0.003 & $<0.01$ \\
\hline \multicolumn{8}{|c|}{ Aging-adjusted data, males } \\
\hline 1959-1985 & 2,997 & -1002 & 0.630 & 0.113 & 5.53 & 0.010 & $<0.00001$ \\
\hline 1963-1985 & 2,539 & -1494 & 0.870 & 0.141 & 6.19 & 0.015 & $<0.00001$ \\
\hline
\end{tabular}

Second, we considered SRTs as a function of birth year, and found a rather steep increase in the general level of about $5 \mathrm{~ms}$ for the four oldest birth years (1959-1962) when compared to the next oldest (1963-1968). This is consistent with significantly slowing auditory SRT in cross-section only from about 50 years of age (Der and Deary, 2006; Jaworski et al., 2011). Participants that represent earlier-born genotypes - expected to have faster reaction times - will also exhibit slower performance due to phenotypic deterioration. Aging effects can in principle be controlled for on the basis of longitudinal studies, which follow the same genotypes as they age. A reasonable estimate of the age-related decline for auditory SRT is an approximately linear increase of $0.5 \mathrm{~ms}$ per year of age from young adulthood to late middle age (Koga and Morant, 1923; Fozard et al., 1994). Those samples were relatively small, about 50-150 individuals per decade, and may therefore not be able to detect non-linearity in the trend. Der and Deary (2006) found, with about 1,000 individuals per decade, both quadratic and cubic trends in visual SRT as a function of age, indicating accelerating decline above about 50 years of age. Any substantial effect of these trends on the SRT magnitude does not seem to appear before 55 years, however (Der and Deary, 2006, pp. 65-66). Thus, the documented aging effects are somewhat ambiguous. The oldest participants in the present sample were 53 years old, and are therefore so close to the suggested breakpoints that decisions to adjust or to exclude cohorts are difficult. We therefore present the data both with and without a linear adjustment of $0.5 \mathrm{~ms}$ per year, and perform regressions both for the full data set and with the four oldest age cohorts $(+50$ years $)$ excluded. This amounts to four distinct trend models.

Figure 1 shows the secular change in SRT across the 27 years, with fitted linear trends for both birth years 1959-1985 and 1963-1985, and for both aging-adjusted and non-adjusted data.

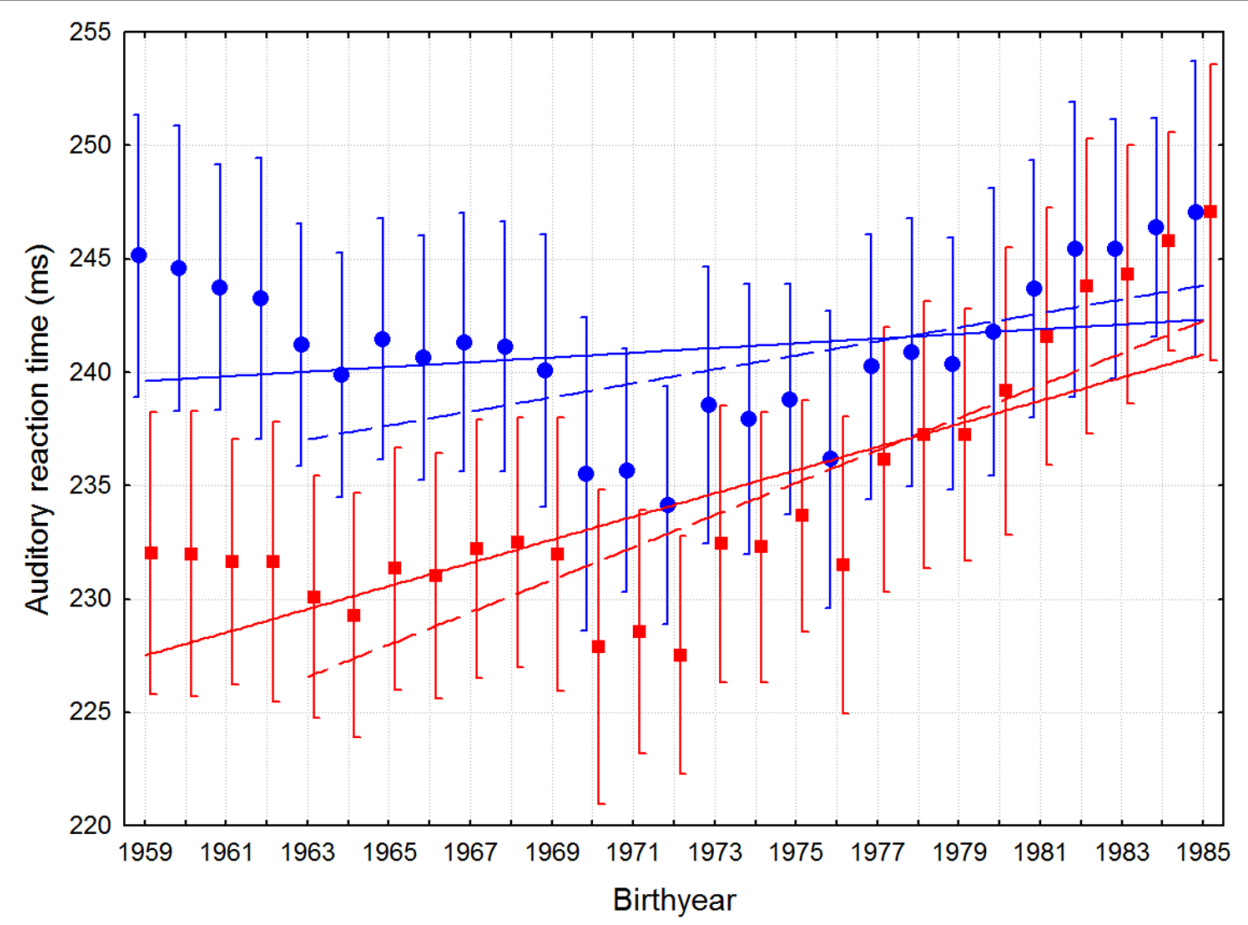

FIGURE 1 | Mean auditory SRT as a function of birth year with 95 percent confidence intervals. The filled circles depict the mean non-adjusted SRTs, and the squares the aging-adjusted SRTs. Linear regression lines are fitted to the data, whose parameters are given in Table 2. 
There are indications of an accelerating slowing from about 1970. Regression analyses, detailed in Table 2, show that, for both sexes together, all temporal trends starting between 1963 and 1985 are significantly different from zero, whereas only the non-adjusted 1959-1985 trend is not.

When separated by sex, the non-adjusted trend for males was significant when the $50+$ participants were excluded, but not for the females. To examine the robustness of the females' nonadjusted trend, regressions were computed for each span from 1959-1985 to 1973-1985, revealing that all trends starting from 1968 or later were significant for the females. With regards to the influence of computer system-related bias, the corresponding increasing trends were somewhat larger for the uncorrected SRT data (see Appendix 1.2).

It could be argued that participants approach the task differently as a function of age, and that this might constitute a measurement error that confounds a real secular slowing in SRT. For example, younger participants might tend to have poorer impulse-control or be more competitive, which would lead them to 'jump the gun.' In this case, such behavior should result in larger performance variability within these cohorts. We therefore computed the SD of SRT across participants in each birth year cohort, which, contrary to predictions from this hypothesis increased with age, from $37 \mathrm{~ms}$ for those born 1985 to $53 \mathrm{~ms}$ for those born 1959 (Appendix Table A1). To summarize this result, the correlation between birth year and SRT SD was -0.318 ( $N=27$, n.s.), consistent with typical effects of aging (Deary and Der, 2005).

It could also be argued that differential attrition as a function of birth year might cause the increase in SRT. This would be the case if there were a tendency for slower SRT individuals to dropout to a greater degree among the older cohorts, perhaps due to factors such as mortality (e.g., Deary and Der, 2005). This possibility was examined by comparing cross-sectional trends among both the participants' and the dropouts' general intelligence test scores. The validity of this approach is based on the fact that less intelligent individuals are more likely to drop out from studies in general (e.g., Deary and Der, 2005, p. 193), and SRT and $g$ are related to one another genetically (Madison et al., in press) and through a common phenotypic chronometric $g$-factor (Verhaeghen and Salthouse, 1997). Scores on the Swedish Enlistment Battery (SEB) during conscription at 19 years of age were used for this purpose. These data were available only for the males who took the test in the interval 19771997, corresponding to birth years 1959-1979. The mean number of individuals per birth year was 127.3 for the participants and 201.0 for the dropouts, with a total of 2,292 participants and 3,619 dropouts. However, the test versions administered in this interval - SEB-67 (1970-1979), SEB-80 (1980-1993), and CATSEB (from 1994) - differ in their structure (Carlstedt, 2000), which incurs a risk of incorrect imputation of secular trends. We therefore performed linear $N$-weighted regressions only for the SEB-80, because it covered the largest numbers of years in the period for which data were available. Significant annual score point increases in intelligence were found among the participants $(\beta=0.085, N=1,739, p<0.00001)$ as well as among the dropouts $(\beta=0.088, N=2,847, p<0.00001)$, as expected based on the Flynn effect (Flynn, 2013). The difference between these trends was however not significant, as the lower confidence interval of the slope for the participants, which was the largest (0.0036), overlapped with the slope for the dropouts. Thus there was no significant tendency for older dropouts to be less intelligent than younger ones, suggesting that attrition is not likely to be generating the observed trends, at least among the male participants and inasmuch as general intelligence serves as a useful proxy for processing speed (e.g., Verhaeghen and Salthouse, 1997; Jensen, 2006).

\section{DISCUSSION}

We found clear trends toward slowing auditory SRT when birth year was regressed against year-on-year SRT means for the years 1959-1985. It is notable that even without adjustment for aging, the SRT speed of the oldest participants is about the same as that of the subsequent generation, whom in the late twenties are supposed to have the shortest SRTs of all age groups (Der and Deary, 2006). Before discussing the implications of these results further, we consider possible confounds and sources of error.

First, online data collection provides much less control than in laboratory measurements. Specifically, we could not assess participants' hearing or fulfillment of the task instructions, although in the case of age-related changes hearing, this would clearly have a suppressing effect on the secular trends observed, in as much as older people have less acute hearing than younger people. Differences in participant computer systems could only partly and indirectly be controlled, by recording their system specifications and comparing results across system components, such as types and versions of operating systems, web browsers, and audio-visual media plug-ins. It is clear that keyboards, sound cards, mice and other hardware components also vary in terms of the lags and performance variances that they add to the speed measurements (Woods et al., 2015). Some post hoc corrections were applied to the SRT data, as described in Appendix 1.2. Whilst they decreased the inter-participant variability, the slowing trend was nonetheless robust across SRT data, both corrected and uncorrected for computer systemrelated variability.

The validity of the SRT data as a measure of general intelligence stems from the finding of a -0.18 correlation with IQ in this sample, despite possible unsystematic error (Mosing et al., 2015). That this correlation is smaller than those values typically found in laboratory studies is consistent with the larger error associated with on-line measurement of both SRT and IQ. The few studies that have correlated auditory SRT task performance with pencil-and-paper measures of IQ have reported correlations between -0.40 to -0.30 , using Raven's SPM (Poon et al., 1986; Agrawal and Kumar, 1993), and -0.05 when the Raven's SPM Plus was employed (Holm et al., 2011). Note that the validity threat in the present study is bias rather than random error, and that a range of possible sources of bias that might be related to birth year were controlled.

Second, longitudinal attrition is a potential source of bias, in as much as there exists a positive correlation between IQ and 
longevity and a positive correlation between IQ and willingness to participate in research. We were however able to assess such effects using conscript IQ data obtained 18-36 years earlier. Older male dropouts did not exhibit lower means of general intelligence than younger ones, neither were their crosssectional characteristics significantly different from those of the participant group. Thus, any true difference between participants and dropouts in terms of a potential age-related decline in $g$ (and by proxy processing speed) is unlikely to have generated the secular increase in SRT. That there was nevertheless a difference which went in the opposite direction makes our estimate of secular SRT increase all the more remarkable. As an additional control, we compared the variability of the dropouts and the participants on the $g$ measure as a means of estimating indirect range restriction, and found that the SD difference was extremely small (0.084) as well as non-significant, despite the large numbers of participants. The difference is nonetheless in the expected direction, that is, larger for the dropouts, but since it is about $1.5 \%$ of the mean SD it must be deemed inconsequential. No correction for restriction of range was therefore performed. Both these calculations were based on males, but it seems reasonable to assume that similar trends would apply for the females, had data been available for them. These calculations rely on the use of the $g$ scores of the attrition sample as a proxy for their SRT scores. If attrition characteristics for different cognitive ability measures are substantially discrepant then this assumption would not hold.

Third, we cannot account for the shorter SRTs for participants born 1970-1971. It could be a continuation of the 1972-1985 trend, followed by aging related increase from 1969-1959, but could also represent some random variability. The regressions are however fitted to all years, except for the 1963 cutoff, and are therefore conservative with respect to the possibly much more dramatic trend suggested in 1972-1985.

Thirdly, we assume that the present sample is representative of the general population. The Swedish Twin Registry (STR) contains all twins born in Sweden, so the remaining issues are whether twins and non-twins or participating and nonparticipating twins differ in some aspect that might interact with age cohort and SRT speed. On the first count, we are not aware of any way in which twins' reaction times would change with birth year in any other way than would be the case for people in general. Indeed, STR data are commonly generalized to the general population across a wide range of variables including, for example, body height and educational attainment (Magnusson et al., 2006), criminality and psychiatric disorders (Webb et al., 2011), and political attitudes (Dawes et al., 2014). On the second count, it has been argued that more genetically similar individuals are also treated similarly, thus violating the equal environments assumption. A large cross-national study using misclassified twins showed the equal environments assumption seems to hold however (Conley et al., 2013).

In the present study, the secular slowing trend was present in all cohort comparisons (males, females, and both sexes combined), and was significant across the entire range of birth years for both the males and the whole sample, but not for the females, who nonetheless exhibited an overall negative trend in SRT performance consistent with potential secular slowing. A test of robustness involved comparing birth years from 1963-1985 for the males and 1973-1985 for the females, and the whole sample, in order to determine the stability of the secular trend. For the whole sample and for the males significant differences were found for all comparisons. For females, significant slowing was observed for birth year ranges from 1968-1985 to 1973-1985, suggesting some trend-robustness also within this subgroup.

A potential cause of the apparent slowing may be exposure to neurotoxic industrial by-products such as heavy metals (Silverman, 2010) and dioxins (ten Tusscher et al., 2014), which may reduce SRT performance via their effects on peripheral nerve conduction velocity. However, as Silverman notes, known neurotoxins have come under tight governmental regulation, emissions have tended to decrease, and serum levels of lead, for example, have decreased since 1970 in the USA (Silverman, 2010, p. 46).

Another possible cause of this trend may be relatively recent micro-evolutionary trends favoring lower $g$ in the population of Sweden. Several studies have revealed that $g$ and fertility are inversely related in the US and the UK (as reviewed in Woodley of Menie, 2015) among cohorts born as far back as the 1890s (Lynn and Van Court, 2004; Lynn, 2011). However, the relationship between $g$ and fertility in Scandinavian countries is less well characterized. Only one study has attempted to examine these trends across birth cohorts in Sweden (Vining et al., 1988). Utilizing aggregate data on fertility and IQ for a mixedsex sample of Swedish cohorts resident in Stockholm county and born between 1909 and 1940 from Vining et al. (1988), it was possible to reconstruct predicted generational changes in genotypic IQ (i.e., the heritable variance component of IQ) due to the changing patterns of selection (i.e., the correlation between IQ and fertility established for each cohort) for four cohorts (see Appendix 2 for details of the method).

Figure 2 reveals considerable inter-cohort variability in the degree to which genotypic IQ would be expected to change per generation, however there is a clear secular trend toward the IQ change becoming increasingly more negative with time

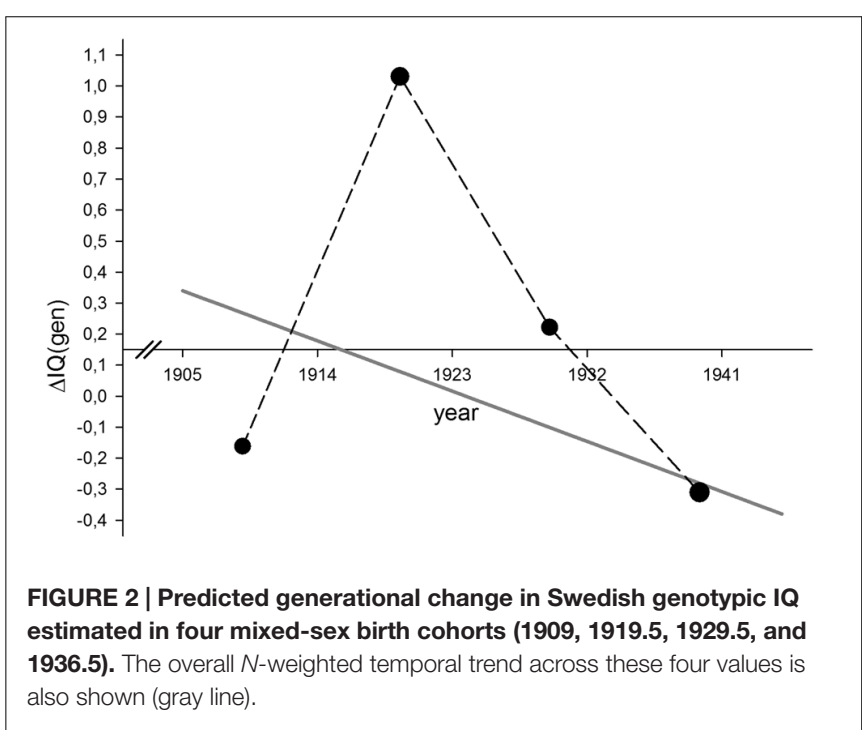


( $r=-0.377, p<0.05, N=1,746)$. This suggests that Sweden transitioned into a selection regime favoring lower levels of genotypic IQ somewhere between 1915 and 1930. The oldest cohorts (those born in the late 1950s) would have had parents born in the late 20 s and early 30 s (assuming a generation length of 2.8-3 decades), thus they would have been born on the cusp of the transition. Subsequent cohorts would have had parents born at a time when microevolutionary trends should have been unambiguously favoring lower genotypic IQ, based on the temporal trend.

Consistent with these observations, Emanuelsson et al. (1993) found evidence that the Flynn effect had leveled off in Swedish cohorts born between 1972 and 1977. A recent study of Swedish 18- to 19-year-old military conscripts evaluated between 1992 and 1993 (born 1973-1974) demonstrates the first indication of an anti-Flynn effect in Sweden, amounting to loss of -0.26 IQ points per decade (Rönnlund et al., 2013). Our finding of slowing SRT could therefore be considered consistent with these findings of stalling Flynn effects and even secular losses among younger Swedish cohorts.

\section{CONCLUSION}

An increasing number of studies converge in indicating secular IQ declines in several geographical regions, using different strands of evidence. The present study has, for the first time, documented a potential secular slowing of SRT in Sweden. More importantly, it employed a measurement design that avoids some of the weaknesses in previous studies as discussed in Dodonova

\section{REFERENCES}

Agrawal, R., and Kumar, A. (1993). The relationship between intelligence and reaction time as a function of task and person variables. Pers. Individ. Dif. 14, 287-288. doi: 10.1016/0191-8869(93)90207-J

Carlstedt, B. (2000). Cognitive Abilities - Aspects of Structure, Process and Measurement. Doctoral thesis, Göteborgs Universitet, Gothenburg, SE.

Conley, D., Rauscher, E., Dawes, C., Magnusson, P. K. E., and Siegal, M. L. (2013). Heritability and the equal environments assumption: evidence from multiple samples of misclassified twins. Behav. Genet. 43, 415-428. doi: 10.1007/s10519013-9602-1

Dawes, C., Cesarini, D., Fowler, J. H., Johannesson, M., Magnusson, P. K. E., and Oskarsson, S. (2014). The relationship between genes, psychological traits, and political participation. Am. J. Pol. Sci. 58, 888-903. doi: 10.1111/ajps.12100

Deary, I. J., and Der, G. (2005). Reaction time, age, and cognitive ability: longitudinal findings from age 16 to 63 years in representative population samples. Aging Neuropsychol. Cogn. 12, 187-215. doi: 10.1080/ 13825580590969235

Der, G., and Deary, I. J. (2006). Age and sex differences in reaction time in adulthood: results from the United Kingdom Health Lifestyle Survey. Psychol. Aging 21, 62-73. doi: 10.1037/0882-7974.21.1.62

Dickinson, M. D., and Hiscock, M. (2011). The Flynn effect in neuropsychological assessment. Appl. Neuropsychol. 18, 136-142. doi: 10.1080/ 09084282.2010.547785

Dodonova, Y. A., and Dodonov, Y. S. (2013). Is there any evidence of historical slowing of reaction time? No, unless we compare apples and oranges. Intelligence 41, 674-687.

Emanuelsson, I., Reuterberg, S. E., and Svensson, A. (1993). Changing differences in intelligence? Comparisons between groups of 13-year-olds tested from 1960 to 1990. Scand. J. Educ. Res. 37, 259-276. and Dodonov (2013). That we essentially replicated previous results provides multi-method validation, which should motivate further study into the reasons for, and possible consequences of, these trends.

\section{AUTHOR CONTRIBUTIONS}

GM and MW conceived the study and wrote the paper, and GM and JS analyzed the data.

\section{FUNDING}

Part of this work was based on a data collection funded by the Bank of Sweden Tercentenary Foundation (M11-0451:1) and the Swedish Scientific Council (HS-2011-1971).

\section{ACKNOWLEDGMENT}

We thank The Swedish Twin Registry for providing the conscript intelligence test data.

\section{SUPPLEMENTARY MATERIAL}

The Supplementary Material for this article can be found online at: http://journal.frontiersin.org/article/10.3389/fnhum. 2016.00407

Flynn, J. R. (2013). The Flynn effect and “Flynn's paradox”. Intelligence 41, 851-857. doi: 10.1016/j.intell.2013.06.014

Fozard, J. L., Vercruysse, M., Reynolds, S. L., Hancock, P. A., and Quiller, R. E. (1994). Age differences and changes in reaction time: the Baltimore longitudinal study of aging. J. Gerontol. 49, 179-189.

Gottfredson, L. S. (2007). Shattering Logic to Explain the Flynn Effect. Cato Unbound. Available at: www.cato-unbound.org/2007/11/08/linda-s-gottfredso $\mathrm{n}$ /shattering-logic-explain-flynn-effect

Holm, L., Ullén, F., and Madison, G. (2011). Intelligence and temporal accuracy of behavior: unique and shared associations between intelligence, reaction time and motor timing. Exp. Brain Res. 214, 175-183. doi: 10.1007/s00221-0112817-6

Jaworski, J., Tchòrzewski, D., and Bujas, P. (2011). Involution of simple and complex reaction times among people aged between 21 and 80 - the results of computer testing. Hum. Mov. 12, 153-158. doi: 10.2478/v10038-0110013-y

Jensen, A. R. (2006). Clocking the Mind: Mental Chronometry and Individual Differences. Amsterdam: Elsevier.

Jensen, A. R. (2011). The theory of intelligence and its measurement. Intelligence 39, 171-177. doi: 10.1016/j.intell.2011.03.004

Koga, Y., and Morant, G. M. (1923). On the degree of association between reaction times in the case of different senses. Biometrika 15, 346-372. doi: $10.2307 / 2331870$

Lichtenstein, P., Floderus, B., Svartengren, M., Svedberg, P., and Pedersen, N. L. (2002). The Swedish Twin Registry: a unique resource for clinical, epidemiological and genetic studies. J. Intern. Med. 252, 184-205. doi: 10.1046/j.1365-2796.2002.01032.x

Lichtenstein, P., Sullivan, P. F., Cnattingius, S., Gatz, M., Johansson, S., Carlström, E., et al. (2006). The Swedish Twin Registry in the third 
millennium: an update. Twin. Res. Hum. Genet. 9, 875-882. doi: 10.1375/twin. 9.6.875

Lynn, R. (2011). Dysgenics: Genetic Deterioration in Modern Populations, 2nd Edn. Ulster: Ulster Institute for Social Research.

Lynn, R., and Van Court, M. (2004). New evidence of dysgenic fertility for intelligence in the united states. Intelligence 32, 193-201. doi: 10.1016/j.intell.2003.09.002

Madison, G., Mosing, M., Verweij, K., Pedersen, N. L., and Ullén, F. (in press). Common genetic influences on intelligence and auditory simple reaction time in a large Swedish sample. Intelligence.

Magnusson, P. K. E., Rasmussen, F., and Gyllensten, U. B. (2006). Height at age 18 years is a strong predictor of attained education later in life: cohort study of over 950,000 Swedish men. Int. J. Epidemiol. 35, 658-663. doi: 10.1093/ije/dyl011

Mosing, M., Madison, G., Pedersen, N. L., Kuja-Halkola, R., and Ullén, F. (2014a). Practice does not make perfect: no causal effect of music practice on music ability. Psychol. Sci. 25, 1795-1803. doi: 10.1177/0956797614541990

Mosing, M., Pedersen, N. L., Madison, G., and Ullén, F. (2014b). Genetic pleiotropy explains associations between musical auditory discrimination and intelligence. PLoS ONE 9:e113874. doi: 10.1371/journal.pone.0113874

Mosing, M., Verweij, K., Madison, G., Pedersen, N. L., Zietsch, B., and Ullén, F. (2015). Did sexual selection shape human music? Testing hypothesis of music evolution using a genetically informative sample of over 10,000 twins. Evol. Hum. Behav. 36, 359-366. doi: 10.1016/j.evolhumbehav.2015.02.004

Nettelbeck, T. J. (2014). Smarter but slower? A comment on Woodley, te Nijenhuis and Murphy (2013). Intelligence 42, 1-4.

Nettelbeck, T. J., and Wilson, C. J. (2004). The Flynn effect: smarter not faster. Intelligence 32, 85-93. doi: 10.1016/S0160-2896(03)00060-6

Panizzon, M. S., Vuoksimaa, E., Spoon, K. M., Jacobson, K. C., Lyons, M. J., Franz, C. E., et al. (2014). Genetic and environmental influences on general cognitive ability: is $g$ a valid latent construct? Intelligence 43, 65-76. doi: 10.1016/j.intell.2014.01.008

Parker, S. (2014). Were the Victorians cleverer than us? Maybe, maybe not. Intelligence 47, 1-2. doi: 10.1016/j.intell.2014.08.002

Pietschnig, J., and Voracek, M. (2015). One century of global IQ gains: a formal metaanalysis of the Flynn effect (1909-2013). Perspect. Psychol. Sci. 10, 282-306. doi: 10.1177/1745691615577701

Poon, P. W. F., Yu, W. Y., and Chan, J. W. C. (1986). Correlation between auditory reaction time and intelligence. Percept. Mot. Skills 63, 375-378. doi: 10.2466/pms.1986.63.2.375

Rönnlund, M., Carlstedt, B., Blomstedt, Y., Nilsson, L.-G., and Weinehall, L. (2013). Secular trends in cognitive test performance: Swedish conscript data 1970-1993. Intelligence 41, 19-24. doi: 10.1016/j.intell.2012.10.001

Silverman, I. W. (2010). Simple reaction time: it is not what it used to be. Am. J. Psychol. 123, 39-50.

Silverman, I. W. (2013). Testing the hypothesized effect of dysgenic fertility on intelligence with existing reaction time data: a comment on Woodley, te Nijenhuis, and Murphy (2013). Intelligence 41, 664-666. doi: 10.1016/j.intell.2013.08.008

Sundet, J. M., Tambs, K., Magnus, P., and Berg, K. (2002). On the question of secular trends in the heritability of intelligence test scores: a study of Norwegian twins. Intelligence 12, 47-59. doi: 10.1016/0160-2896(88)90022-0

ten Tusscher, G. W., Leijs, M. M., de Boer, L. C, Legler, J., Olie, K., Spekreijse, H., et al. (2014). Neurodevelopmental retardation, as assessed clinically and with magnetoencephalography and electroencephalography, associated with perinatal dioxin exposure. Sci. Total Environ. 491-492, 235-239. doi: 10.1016/j.scitotenv.2014.02.100

Theorell, T., Lennartsson, A.-K., Madison, G., Mosing, M. A., and Ullén, F. (2015). Predictors of continued playing or singing - from childhood and adolescence to adult years. Acta Pediatr. 104, 274-284. doi: 10.1111/apa.12870

Ullén, F., Mosing, M., Holm, L., Eriksson, H., and Madison, G. (2014). Psychometric properties and heritability of a new online test for musicality, the Swedish Musical Discrimination Test. Pers. Individ. Dif. 63, 87-93. doi: 10.1016/j.paid.2014.01.057
Ullén, F., Mosing, M., and Madison, G. (2015). Associations between motor timing, music practice, and intelligence studied in a large sample of twins. Ann. N. Y. Acad. Sci. 1337, 125-129. doi: 10.1111/nyas.12630

Verhaeghen, P. (2014). The Elements of Cognitive Aging: Meta-analyses of Aggregated Differences in Processing Speed and their Consequences. Oxford: Oxford University Press.

Verhaeghen, R., and Salthouse, T. A. (1997). Meta-analyses of age-cognition relations in adulthood: estimates of linear and nonlinear age effects and structural models. Psychol. Bull. 122, 231-249. doi: 10.1037/00332909.122.3.231

Vining, D. R. Jr., Bygren, L., Hattori, K., Nystrom, S., and Tamura, S. (1988). IQ/fertility relationships in Japan and Sweden. Pers. Individ. Dif. 9, 931-932. doi: 10.1016/0191-8869(88)90015-3

Wallace, A., and Madison, G. (2012). The timing accuracy of general purpose computers for experimentation and measurements in psychology and the life sciences. Open Psychol. J. 5, 44-53. doi: 10.2174/1874350101205010044

Webb, R. T., Långström, N., Runeson, B., Lichtenstein, P., and Fazel, S. (2011). Violent offending and IQ level as predictors of suicide in schizophrenia: national cohort study. Schizophr. Res. 130, 143-147. doi: 10.1016/j.schres.2011.04.031

Wicherts, J. M., Dolan, C. V., Hessen, D. J., Oosterveld, P., van Baal, G. C. M., Boomsma, D. I., et al. (2004). Are intelligence tests measurement invariant over time? Investigating the nature of the Flynn effect. Intelligence 32, 509-537.

Woodley, M. A., Madison, G., and Charlton, B. G. (2014a). Possible dysgenic trends in simple visual reaction time performance in the Scottish Twenty-07 cohort: a reanalysis of Deary \& Der (2005). Mankind Q. 55, 110-124.

Woodley, M. A., te Nijenhuis, J., and Murphy, R. (2014b). Is there a dysgenic secular trend towards slowing simple reaction time? Responding to a quartet of critical commentaries. Intelligence 46, 131-147. doi: 10.1016/j.intell.2014. 05.012

Woodley, M. A., te Nijenhuis, J., and Murphy, R. (2013). Were the Victorians cleverer than us? The decline in general intelligence estimated from a metaanalysis of the slowing of simple reaction time. Intelligence 41, 843-850. doi: 10.1016/j.intell.2013.04.006

Woodley of Menie, M. A. (2015). How fragile is our intellect? Estimating losses in general intelligence due to both selection and mutation accumulation. Pers. Individ. Dif. 75, 80-84. doi: 10.1016/j.paid.2014.10.047

Woodley of Menie, M. A., and Fernandes, H. B. F. (2016). Showing their true colours: possible secular declines and a Jensen effect on color acuity - more evidence for the weaker variant of Spearman's other hypothesis. Pers. Individ. Dif. 88, 280-284. doi: 10.1016/j.paid.2015.09.009

Woodley of Menie, M. A., te Nijenhuis, J., and Murphy, R. (2015a). Are signal luminances and confounded stimuli a source of slowing simple reaction time and cross-study heterogeneity? A response to Parker (2014). Intelligence 49, 23-24. doi: 10.1016/j.intell.2014.12.003

Woodley of Menie, M. A., te Nijenhuis, J., Murphy, R. (2015b). The Victorians were still faster than us. Commentary: factors influencing the latency of simple reaction times. Front. Hum. Neurosci. 9:452. doi: 10.3389/fnhum.2015. 00452

Woods, D. L., Wyma, J. M., Yund, E. W., Herron, T. J., and Reed, B. (2015). Factors influencing the latency of simple reaction time. Front. Hum. Neurosci. 9:131. doi: 10.3389/fnhum.2015.00131

Conflict of Interest Statement: The authors declare that the research was conducted in the absence of any commercial or financial relationships that could be construed as a potential conflict of interest.

Copyright (c) 2016 Madison, Woodley of Menie and Sänger. This is an open-access article distributed under the terms of the Creative Commons Attribution License (CC BY). The use, distribution or reproduction in other forums is permitted, provided the original author(s) or licensor are credited and that the original publication in this journal is cited, in accordance with accepted academic practice. No use, distribution or reproduction is permitted which does not comply with these terms. 